\title{
Insufficient ascorbic acid intake during gestation induces abnormal cardiac dilation in fetal and neonatal SMP30/GNL knockout mice
}

\author{
Yuki Kishimoto, ${ }^{1,2}$, Takao Kanai ${ }^{3}$, Kayoko Sato ${ }^{4}$, Jaewon Lee ${ }^{5}$, Kyu-Shik Jeong ${ }^{6}$, Kentaro Shimokado ${ }^{2}$, Naoki Maruyama \\ and Akihito Ishigami ${ }^{1}$
}

BACKGROUND: Despite the acknowledged importance of ascorbic acid (AA) in maintaining pregnancy and normal fetal development, its precise actions remain obscure. Therefore, we investigated the impact of maternal AA content on the growth of fetal mice during the gestation period using senescence marker protein-30/gluconolactonase (SMP30/GNL) knockout $(\mathrm{KO})$ mice, which cannot synthesize AA in vivo.

METHODS: SMP30/GNL KO mice gave birth after a gestation period under conditions of absent, low, or normal AA intake. AA was measured using high-performance liquid chromatography and electrochemical detection. Whole-body sections were stained with hematoxylin and eosin, Elastica van Gieson, and Azan.

RESULTS: The mothers in the group absent AA intake failed to bear young because of incomplete fetal development. Offspring born under the low-AA condition generally died within a few days after birth. Morphological analysis revealed that the latter neonates of SMP30/GNL KO mothers whose intake of AA was low during gestation manifested abnormal cardiac dilation, congestion of the liver and lungs, incompletely expanded pulmonary alveoli, and impaired vertebral bodies. In contrast, a normal AA diet produced healthy progeny.

CONCLUSION: A diet sufficiently replete with AA is essential during the gestational period for normal tissue development in the fetus and neonate.

A scorbic acid (AA) has numerous metabolic functions that are largely dependent on its potent toxin-reducing properties (1). AA acts as a cofactor in reactions catalyzed by several metal-dependent oxygenases, such as $\mathrm{Cu}^{+}$-dependent monooxygenases including peptidylglycine $\alpha$-amidating monooxygenase involved in peptide hormone synthesis $(2,3)$; dopamine $\beta$-hydroxylase, a component of norepinephrine synthesis $(4,5)$; and $\mathrm{Fe}^{2+} / \alpha$-ketoglutarate-dependent dioxygenases including prolyl and lysyl hydroxylases, which stabilize the triple helical structure of collagen (6,7). Moreover, AA functions as an electron donor and scavenges free radicals such as superoxide radicals (8) and hydroxyl radicals (9).

Humans and guinea pigs fail to synthesize AA in vivo because they lack L-gulonolactone oxidase, the enzyme that catalyzes the last step of the AA synthesis pathway (10). Similarly, the ailment termed osteogenic disorder shionogi in rats and Gulo knockout (KO) mice, which also lack gulonolactone oxidase, stems from their failure to synthesize AA in vivo $(11,12)$. A recent report described aldehyde reductase and aldose reductase double-KO mice that were unable to synthesize AA and developed scurvy (13). Aldehyde reductase and aldose reductase catalyze the conversion of D-glucuronic acid to L-gulonic acid, the enzyme of the antepenultimate step of the AA synthesis pathway. Therefore, these animals must obtain AA from dietary sources.

Senescence marker protein-30 (SMP30) is a $34-\mathrm{kDa}$ protein whose expression in the liver, kidney, and lung decreases with age in an androgen-independent manner $(14,15)$. Previously, we revealed that SMP30 was a gluconolactonase (GNL) responsible for the conversion of L-gulonic acid to L-gulono- $\gamma$-lactone, the enzyme of the penultimate step of the AA synthesis pathway (16). In addition, SMP30/GNL KO mice developed symptoms of scurvy when fed an AA-free diet. Therefore, SMP30/GNL $\mathrm{KO}$ mice are extremely valuable for investigating the physiological functions of AA in vivo.

$\mathrm{AA}$ is thought to be required for the maintenance of pregnancy and fetal development. However, the effects of the absence of AA on embryonic and neonatal development during pregnancy have not been determined, with the exception of a reported detriment to brain development $(17,18)$. Therefore, we have examined pregnant mice completely or partially deprived of AA to test embryonic and neonatal development. In SMP30/GNL KO mice, the absence of AA resulted in the death of embryos in utero, and a low intake of AA during gestation induced readily apparent abnormal tissue development in newborns.

\footnotetext{
'Molecular Regulation of Aging, Tokyo Metropolitan Institute of Gerontology, Tokyo, Japan; ${ }^{2}$ Department of Geriatrics and Vascular Medicine, Tokyo Medical and Dental University, Tokyo, Japan; ${ }^{3}$ nstitute of Laboratory Animals, Tokyo Women's Medical University, Tokyo, Japan; ${ }^{4}$ Department of Cardiology, Tokyo Women's Medical University, Tokyo, Japan; ${ }^{5}$ Department of Pharmacy, Pusan National University, Busan, Republic of Korea; ${ }^{6}$ Department of Pathology, Kyungpook National University, Daegu, Republic of Korea. Correspondence: Akihito Ishigami (ishigami@tmig.or.jp) 


\section{RESULTS}

Effects of a Maternal Absence of AA on the Fetus During Gestation After mating SMP30/GNL KO males and females, we confirmed the latter's pregnancy by the presence of a vaginal plug. These females were divided into a normal AA $($ AA $(+))$ group and an absent AA (AA (-)) group (Figure 1a). The AA $(+)$ group had free access to water containing $1.5 \mathrm{~g} / 1 \mathrm{AA}$, whereas the AA (-) group was given water without AA. AA (-) mothers tested at the start of gestation (day 0) had plasma AA levels of $61.9 \pm 4.4 \mu \mathrm{mol} / \mathrm{l}$ (Figure 1b). However, at days 5, 10, and 15 of pregnancy, this group's AA levels in plasma decreased significantly to $14.7 \pm 2.0,5.3 \pm 1.0$, and $1.8 \pm 0.3 \mu \mathrm{mol} / \mathrm{l}$, respectively. Moreover, by day 20 of pregnancy, the AA (-) SMP30/ GNL KO mice had no detectable AA in their plasma. On the contrary, the AA levels in plasma of AA (+) SMP30/GNL KO mice were not different from those of days 0 to 15 of pregnancy, although the difference became statistically significant when days 0 and 20 of pregnancy were compared (Figure $1 \mathbf{b}$ ).

The maternal body weight of each SMP30/GNL KO mouse on the first gestational day (day 0 ) was $21.4 \pm 0.6 \mathrm{~g}$. These AA $(-)$ mothers increased in weight from day 0 to 15 of pregnancy and then decreased from gestational days 15 to 20 (Figure 1c). On the other hand, AA (+) SMP30/GNL KO mothers gained weight throughout their $20 \mathrm{~d}$ of pregnancy. Furthermore, the difference in body weight between AA (+) and AA (-) SMP30/ GNL KO females was significant at day 20 of pregnancy (Figure 1c).

The young of AA (+) SMP30/GNL KO mice were born and matured normally. However, none of the fetal mice from AA (-) SMP30/GNL KO mice achieved live birth, even after the expected
$20 \mathrm{~d}$ of pregnancy. To establish the cause, some uteri were extirpated from AA (-) SMP30/GNL KO mice on day 19 prepartum. All fetuses were already dead, but even then their development was incomplete (Figure 1d). In stark contrast, during the same time period all fetuses of AA (+) SMP30/GNL KO mice were alive and normal (Figure 1e). AA (-) SMP30/GNL KO mice carried significantly fewer fetuses (mean litter size $5.3 \pm 0.6$ ) on prepartum day 19 than AA (+) SMP30/GNL KO mice (mean litter size $8.4 \pm 1.2$ ), as depicted graphically in Figure 1f.

\section{Effect of Low AA on the Fetus During Gestation}

Because the complete lack of AA during gestation resulted in embryonic lethality, we next examined the effect of low AA intake on fetal development. Female SMP30/GNL KO mice were divided into two groups: normal AA (AA (+)) recipients of $1.5 \mathrm{~g} / \mathrm{l} \mathrm{AA}$ in water and low AA (AA (Low)) recipients of $0.0375 \mathrm{~g} / \mathrm{l} \mathrm{AA}$ ad libitum (Figure 2a). The plasma of AA (Low) SMP30/GNL KO mice at the start of gestation (day 0) contained $2.7 \pm 0.7 \mu \mathrm{mol} / \mathrm{l} \mathrm{AA}$, which was $<5 \%$ of that for AA (+) SMP30/GNL KO mice, and on day 20 of pregnancy this former group (Low) had virtually no AA in their plasma (i.e., only $0.5 \pm 0.1 \mu \mathrm{mol} / \mathrm{l}$ ) (Figure 2b).

All AA (+) and AA (Low) SMP30/GNL KO mice gave birth (Figure 2c-e). Respiratory failure, systemic bleeding (Figure 2d), and anophthalmia (Figure 2e) were observed in some of the neonates from AA (Low) SMP30/GNL KO mice, but none of the AA (+) SMP30/GNL KO mice had these abnormalities (Figure 2c). The percentages of respiratory failure, systemic bleeding, and anophthalmia of neonates from AA (Low) SMP30/GNL KO mice were 15.7, 11.8, a

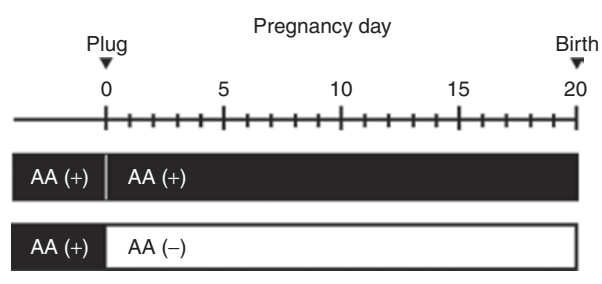

d

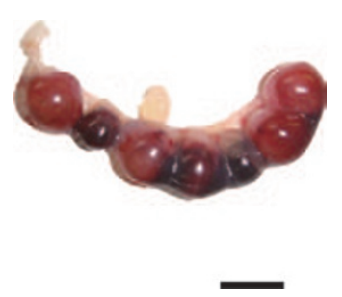

b

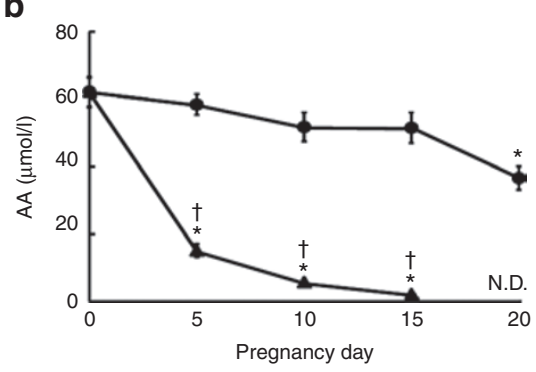

C

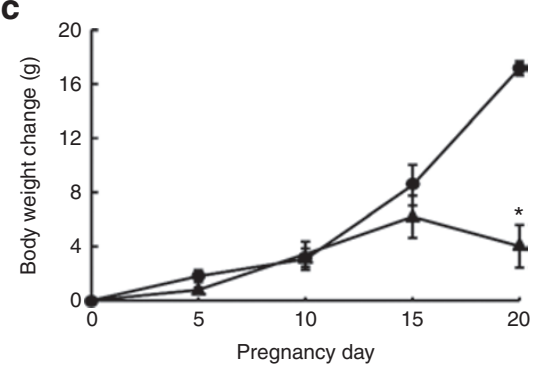

e

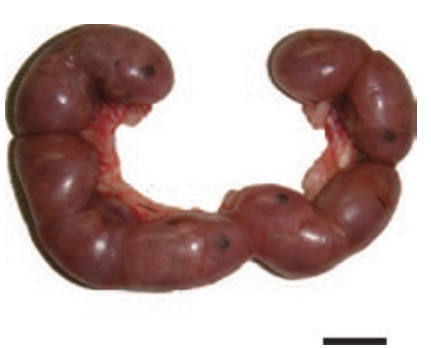

f

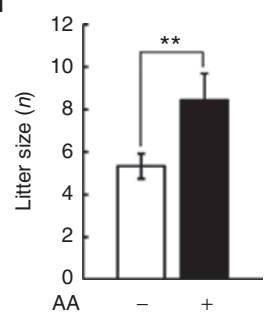

Figure 1. Effect of absent AA during gestation. (a) The pregnant female SMP30/GNL KO mice were divided into normal (AA (+)) and absent AA (AA (-)) groups. (b) Plasma AA levels of these AA $(+)$ mothers are indicated by black-filled circles and those of $A A(-)$ mothers by black-filled triangles. ${ }^{*} P<0.01$ between day 0 and days $5,10,15$, and 20 of pregnancy. ${ }^{\dagger} P<0.01$, between $A A(+)$ and AA ( - ) at the same day of pregnancy. (c) Body weights of AA ( + ) mothers are indicated by black-filled circles and those of $A A(-)$ mothers by black-filled triangles. ${ }^{*} P<0.01$ at day 20 of pregnancy. (d, e) Appearance of uteri extirpated on day 19 of pregnancy from (d) AA (-) and (e) AA (+) SMP30/GNL KO mice. Bars $=1 \mathrm{~cm}$. In a-e, values are expressed as means \pm SEM of 10 animals. (f) The mean litter size of AA (-) and AA (+) SMP30/GNL KO mice at day 19 of pregnancy. Values are expressed as means \pm SEM of 64 AA ( - ) and $76 \mathrm{AA}(+)$ SMP30/GNL KO animals. ${ }^{* *} P<0.05$. AA, ascorbic acid; GNL, gluconolactonase; KO, knockout; N.D., not detected; SMP, senescence marker protein. 

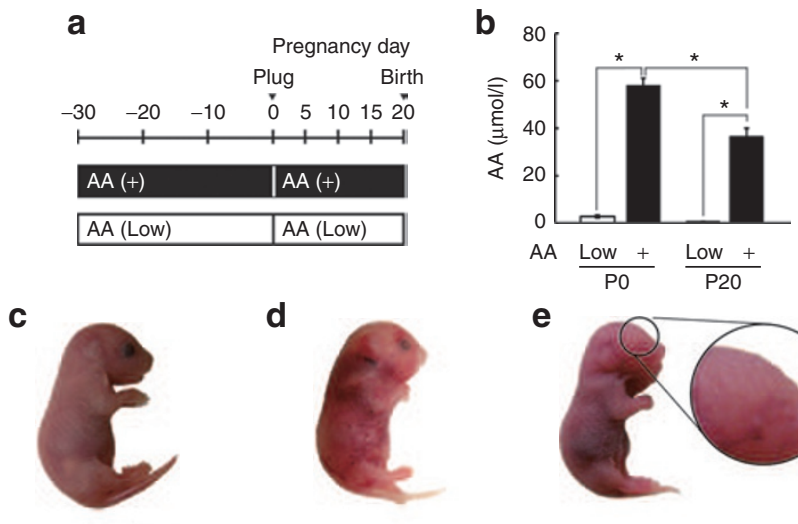

d

f
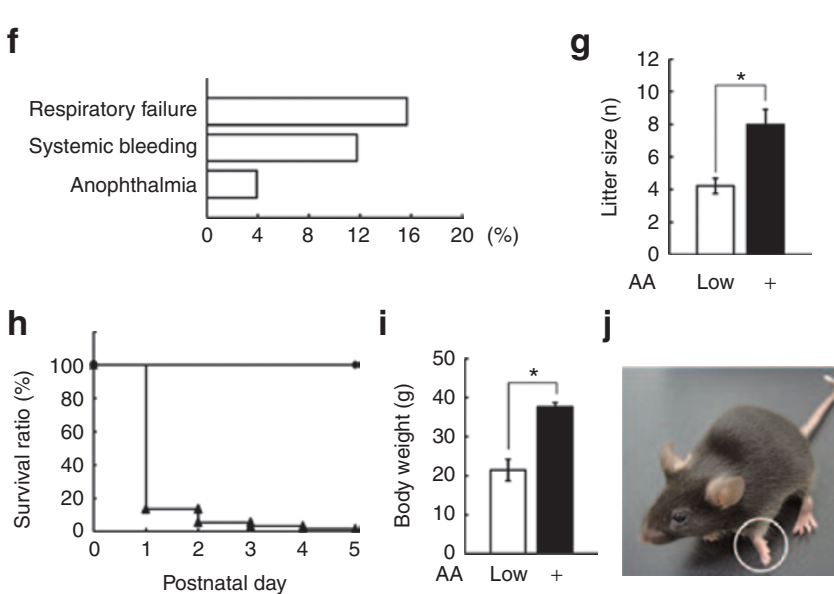

j
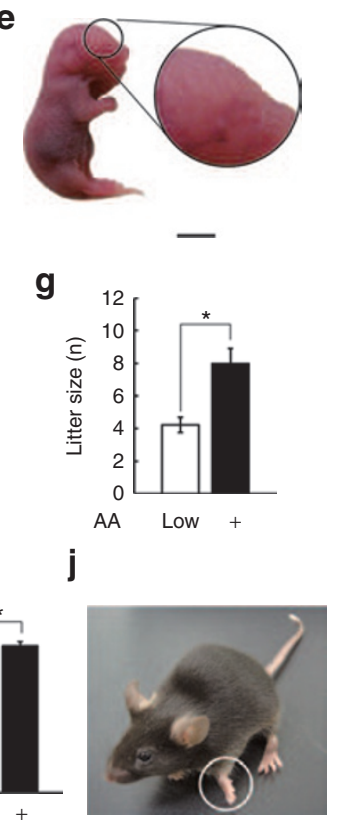

Figure 2. Effect of low $A A$ on the fetus during gestation. (a) Before mating, female SMP30/GNL KO mice were divided into AA (+) and AA (Low) groups. (b) The plasma AA levels of AA (+) and AA (Low) SMP30/GNL KO females on the day before mating (P0) and at day 20 of pregnancy (P20). Values are expressed as means \pm SEM of 10 animals. ${ }^{*} P<0.01$. (c, d, e) Appearance of neonates from (c) AA (+) and (d, e) AA (Low) SMP30/GNL KO mice.

Bars $=5 \mathrm{~mm}$. (f) Developmental abnormalities were recorded in a total of 51 neonates from AA (Low) SMP30/GNL KO mothers. Respiratory failure in these offspring indicates discontinuation of spontaneous breathing. (g) The mean litter size of AA (Low) and AA (+) SMP30/GNL KO mice at day 20 of pregnancy. Values are expressed as means \pm SEM of 119 AA (Low) and 102 AA (+) SMP30/GNL KO animals. ${ }^{*} P<0.01$. (h) Survival curves of AA (Low) and AA (+) SMP30/GNL KO mice. The numbers of living neonates of AA (+) mothers, as indicated by black-filled circles, were 204 at postnatal day 5 . The numbers of living neonates of the AA (Low) group, as indicated by blackfilled triangles, were 184 (day 0), 25 (day 1), 10 (day 2), 6 (day 3), and 3 (day 4) for each postnatal day. (i) Body weights of AA (Low) and AA (+) SMP30/GNL $\mathrm{KO}$ mice at 5 mo of age. Values are expressed as means \pm SEM of three AA (Low) and five AA (+) SMP30/GNL KO animals. ${ }^{*}<0.01$. (j) A few surviving young from AA (Low) SMP30/GNL KO mothers had dysostosis at 3 mo of age, as indicated with a white circle, and died by 12 mo of age. AA, ascorbic acid; GNL, gluconolactonase; KO, knockout; SMP, senescence marker protein.

and 3.9\%, respectively (Figure 2f). Moreover, the litter sizes of neonates from AA (Low) SMP30/GNL KO mice were significantly smaller than those from AA (+) SMP30/GNL KO mice (Figure 2g). Most neonates from AA (Low) SMP30/ GNL KO mice died within several days after birth (Figure $2 h$ ), leaving only a few that survived and grew to adolescence. Of those survivors, all were smaller in stature than their AA (+) counterparts by the age of 5 mo (Figure 2i), showed dysostosis (Figure $2 \mathbf{j}$ ), and died by 12 mo of age.
Whole-Body and Tissue Disparities of Fetal Mice After Day 19 of Pregnancy

To investigate the effect of low AA and avoid the influence of breastfeeding on neonatal development, some fetal mice were extirpated at prenatal day 19 , just before birth, from the AA (+) and AA (Low) SMP30/GNL KO mice. At that time, both the body weights and the body lengths differed significantly between the fetuses from AA (+) and AA (Low) SMP30/GNL KO mice (Table 1). Moreover, the cardiac tissues of fetal AA (Low) mice weighed significantly less than those of the AA (+) mice, although the lungs and liver of the two groups did not significantly differ in weight (Table 1).

\section{AA Content of Whole Body and Tissues in the Fetal Mice at Day 19 of Pregnancy}

The AA levels of whole body, heart, lung, and liver in the fetal mice from AA (Low) SMP30/GNL KO mice were $<10 \%$ of that in the matched AA (+) SMP30/GNL KO mice (Table 1).

\section{Morphological Analysis of Neonatal Mice Within 24h After Birth}

To examine further the effect of low AA on fetal development, we prepared whole-body sections of neonates from the AA $(+)$ and AA (Low) groups within $24 \mathrm{~h}$ after birth and stained the tissues with hematoxylin and eosin. Abnormalities were notable in numerous tissues including the heart, lung, liver, and vertebral column of infants from AA (Low) SMP30/GNL KO mice as compared with those from AA (+) SMP30/GNL KO mice (Figure 3a,b). In the liver, congestion was marked in the AA (Low) SMP30/GNL KO neonates (Figure 3c,e), but not in their AA (+) SMP30/GNL KO counterparts (Figure 3d,f). In the heart, the epicardium was significantly larger in AA (Low) SMP30/GNL KO mice $(9.6 \pm 0.1 \mathrm{~mm})$ than in AA (+) SMP30/GNL KO mice $(8.5 \pm 0.2 \mathrm{~mm}$ ) (Figure $4 \mathbf{a}, \mathbf{b}, \mathbf{e})$. At the chest side, apex, and back side of the neonates' myocardium, AA (Low) SMP30/GNL KO fetal mice had significantly thinner dimensions than those found in the matched AA (+) group (Figure 4a-c,d,f). Thinning of the AA (Low) group's myocardial wall at the chest side was evident in a microscopic high-power field as compared with samples from AA (+) SMP30/GNL KO mice (Figure $4 \mathbf{c}, \mathbf{d}$ ). Moreover, elastin fiber composed of arterial media was configured loosely in the neonatal mice from AA (Low) SMP30/GNL KO dams (Figure 4g). However, elastin fibers of the aortic wall were assembled properly in the neonates from AA (+) SMP30/GNL KO mothers (Figure 4h).

The lungs were notably congested in neonatal mice from AA (Low) SMP30/GNL KO mice (Figure 5a) but not AA (+) stock (Figure $5 \mathrm{~b}$ ). To evaluate the size of pulmonary airspaces, we measured the mean linear intercepts. At $48.1 \pm 1.6 \mu \mathrm{m}$, the mean linear intercepts from AA (Low) SMP30/GNL KO mice were significantly smaller than those from AA (+) SMP30/ GNL KO mice (Figure 5c), which were $76.5 \pm 2.8 \mu \mathrm{m}$.

In the thoracic vertebral column, we noted dramatic impairment of vertebral bodies, which contained a core of hypertrophic chondrocytes flanked with prehypertrophic cells and proliferating chondroblasts in AA (Low) SMP30/GNL KO mice but not in their AA (+) partners (Figure 6a,b). 
Table 1. Physical characteristics and AA levels of fetal mice

\begin{tabular}{lrc}
\hline & AA (+) & AA (Low) \\
\hline Body weight $(\mathrm{g})$ & $1.2 \pm 0.1$ & $1.0 \pm 0.1^{* *}$ \\
Body length $(\mathrm{mm})$ & $37.4 \pm 0.4$ & $35.1 \pm 0.4^{*}$ \\
Tissue weight $(\mathrm{mg})$ & & \\
$\quad$ Heart & $6.9 \pm 0.6$ & $5.0 \pm 0.3^{* *}$ \\
Lung & $37.3 \pm 1.7$ & $32.7 \pm 1.2$ \\
Liver & $53.6 \pm 3.7$ & $50.9 \pm 2.6$ \\
AA ( $\mu$ mol/g weight) & & \\
Whole body & $1.29 \pm 0.02$ & $0.13 \pm 0.02^{*}$ \\
Heart & $0.30 \pm 0.04$ & $0.03 \pm 0.00^{*}$ \\
Lung & $0.98 \pm 0.13$ & $0.07 \pm 0.00^{*}$ \\
Liver & $0.42 \pm 0.03$ & $0.03 \pm 0.00^{*}$ \\
\hline
\end{tabular}

Fetal mice were extirpated at day 19 of pregnancy from the AA (+) and AA (Low) SMP30/GNL KO mice. Values are expressed as means \pm SEM of 10 animals.

AA, ascorbic acid; GNL, gluconolactonase; KO, knockout; SMP, senescence marker protein

${ }^{*} P<0.01$ and ${ }^{*} P<0.05$. a

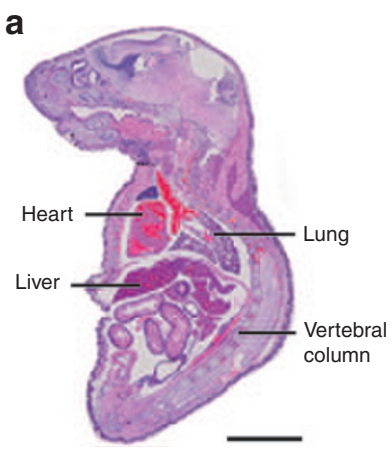

C

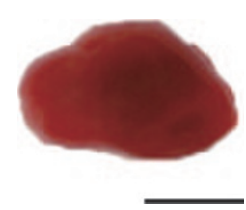

e

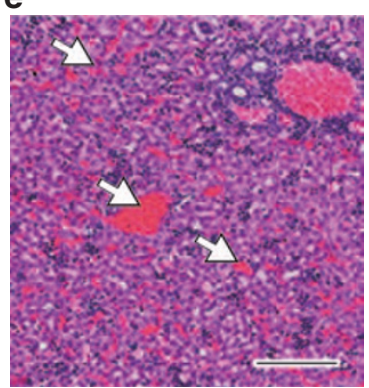

b

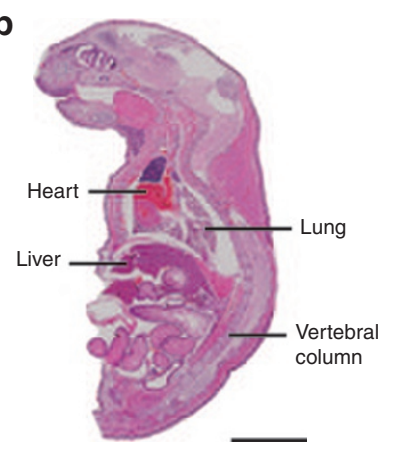

d
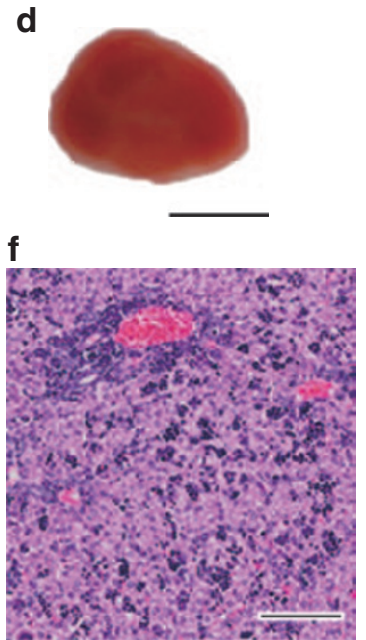

Figure 3. Morphological analysis of mice within $24 \mathrm{~h}$ after birth. Representative whole-body sections of neonates from (a) AA (Low) and (b) AA (+) SMP30/GNL KO females. Bars = $2 \mathrm{~mm}$. Appearance of liver extirpated from neonates of (c) AA (Low) and (d) AA (+) SMP30/GNL KO females. Bars $=0.5 \mathrm{~cm}$. Representative liver section of neonates from (e) AA (Low) and (f) AA (+) SMP30/GNL KO mice. White arrows indicate the typical appearance of congestion. Bars $=100 \mu \mathrm{m}$. AA, ascorbic acid; GNL, gluconolactonase; KO, knockout; SMP, senescence marker protein.

\section{DISCUSSION}

In the present study, we examined the impact of maternal AA consumption on fetal development by comparing two conditions of the vitamin's intake and three outcomes. The SMP30/ GNL KO mice used in this study lack the capacity to synthesize AA in vivo, thereby providing a useful model for analyzing environments of absent AA or low AA vs. normal AA. In the absence of AA, SMP30/GNL KO mothers did not produce live births even after the expected full term; that is, all their fetuses died in utero because of incomplete development during the gestational period. On the other hand, all SMP30/GNL KO mothers with a minimal AA intake (AA (Low)) bore live progeny, but we found that the mothers' inadequate intake of $\mathrm{AA}$, caused by a diet low in AA given for $30 \mathrm{~d}$ prior to and during gestation, yielded offspring with abnormal cardiac dilation, congestion of the liver and lungs, incompletely expanded pulmonary alveoli, and impaired vertebral bodies. In fact, this is the first report to recognize an association between a low AA intake and abnormal heart development.

In the state of absent AA, the SMP30/GNL KO mothers had significantly decreased plasma AA levels during gestation. At day 5 of pregnancy, their plasma AA levels were already $24 \%$ lower than at the start of gestation (day 0). Subsequently, the plasma AA levels decreased dramatically and finally became undetectable at day 20 of pregnancy. Similarly, others reported that the plasma AA levels of humans were depleted $4 \mathrm{wk}$ after ingesting an AA-free diet (6). In normal AA (i.e., AA (+)) SMP30/GNL KO dams, plasma AA levels had decreased slightly during gestational days 0 to 15 , yet at day 20 of pregnancy dropped to a significant $38 \%$ lower than on gestational day 0 . In dramatic contrast, the body weights of fetuses born to AA $(+)$ mothers increased markedly during late gestation; therefore, AA must be necessary to produce a normal skeletal structure during fetal development.

In the group of SMP30/GNL KO mothers given a minimal AA (i.e., AA (Low)) diet for 30 prenatal days, the plasma levels of AA were $<5 \%$ of that in their AA $(+)$ counterparts. Moreover, at $20 \mathrm{~d}$ of pregnancy, the barely detectable levels of AA in the plasma of these AA (Low) mice were $<1.4 \%$ of the content in AA (+) mice. Still, these maternal plasma AA levels were enough to prevent an embryonic demise, so that neonates of AA (Low) SMP30/ GNL KO mice were born alive, although most died within a few days. Moreover, some of the young had extensive internal hemorrhages and eye abnormalities such as microphthalmia and anophthalmia. Clearly, AA has an important role in angiogenesis and eye development during the gestational period. Accordingly, Sotiriou et al. (19) reported that sodium-dependent vitamin C transporter $2 \mathrm{KO}$ mice, which lacked an AA transporter, died from respiratory failure and diffuse cerebral hemorrhage within a few minutes of birth. Thus, AA is essential not only for prenatal normality but also for perinatal survival.

Many studies have been carried out to elucidate the role of AA in brain development. In one such study, the AA concentration in the human forebrain at 11-19 wk of gestation proved to be 4-11 times higher than that of adults (20). Qiu et al. (17) reported that cultured hippocampal neurons from fetal 


\section{Articles $\mid$ Kishimoto et al.}

a
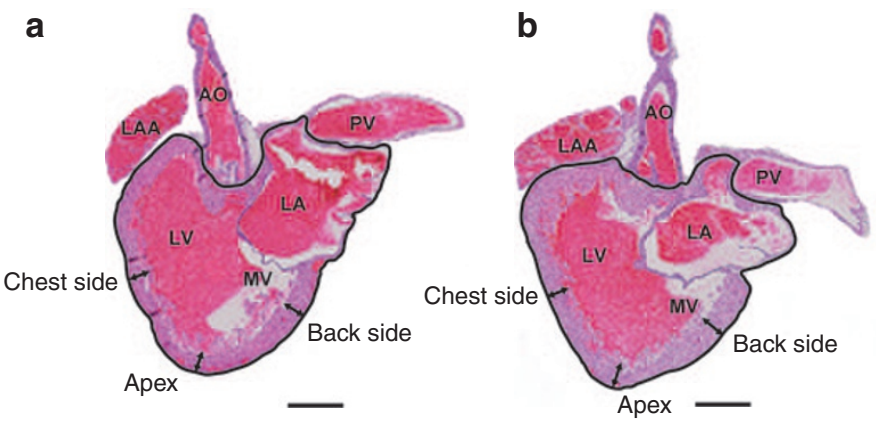

C
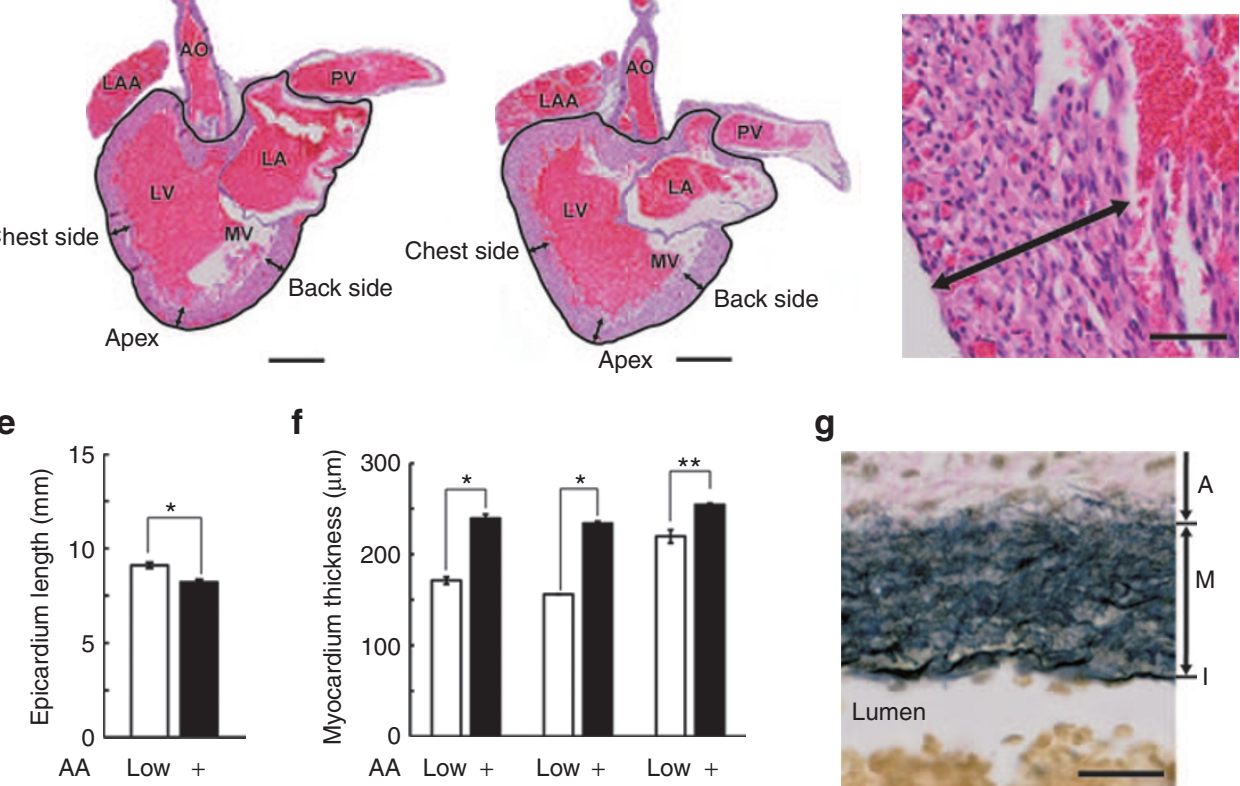

f

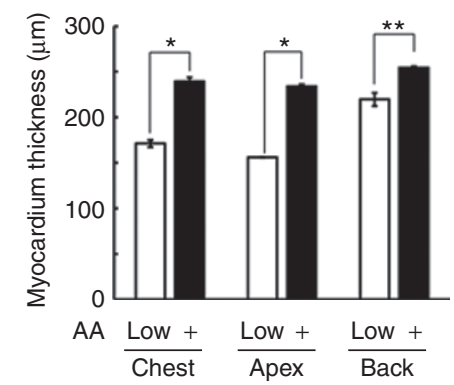

g

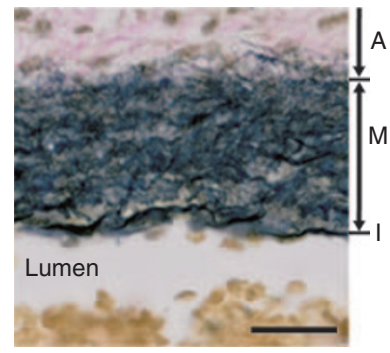

d

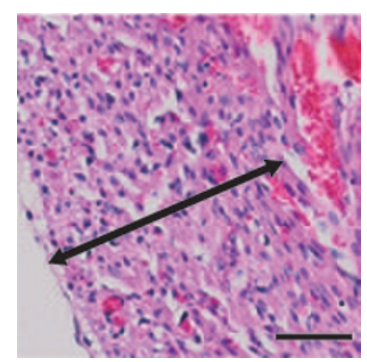

h

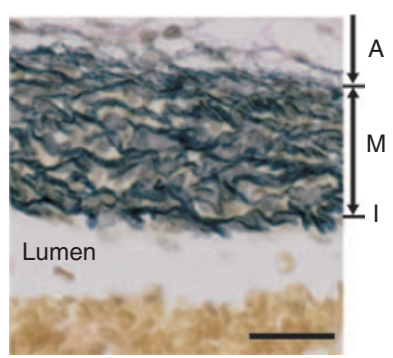

Figure 4. Uniform sagittal plane sections of a heart $(\mathbf{a}, \mathbf{b})$ include the left atrium (LA), left ventricle (LV), left atrial appendage (LAA), pulmonary vein (PV), aorta (AO), mitral valve (MV), and apex. Representative heart section of neonates from (a) AA (Low) and (b) AA (+) SMP30/GNL KO mice. The black line depicts the edge of the heart. Bars $=500 \mu \mathrm{m}$. (c, d) High-power field of LV walls at the chest side from (c) AA (Low) and (d) AA (+) SMP30/GNL KO mice. Black two-directional arrows indicate thickness of the myocardium. Bars $=50 \mu \mathrm{m}$. Panels $\mathbf{e}$ and $\mathbf{f}$ portray the epicardial length of the heart (e) and thickness of myocardium at the chest side, apex, and back side of the LV (f), measured as indicated in a-d. Values are expressed as means \pm SEM of 10 animals. ${ }^{*} P<0.01$ and ${ }^{*} P<0.05$. Uniform sagittal plane sections of AO stained with Elastica van Gieson from (g) AA (Low) and (h) AA (+) SMP30/GNL KO mice. Bars $=25 \mu \mathrm{m}$. A, arterial adventitia; AA, ascorbic acid; I, arterial intima; KO, knockout; M, arterial media; SMP, senescence marker protein.

a

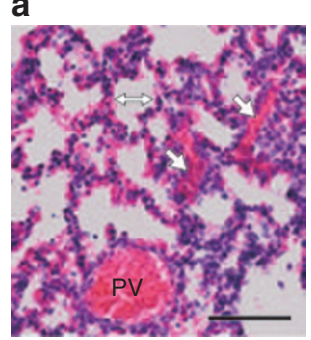

b

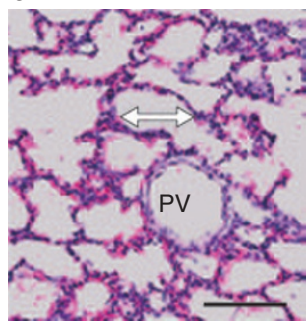

C

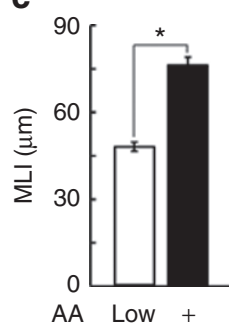

Figure 5. Representative lung sections of neonatal mice from (a) AA (Low) and (b) AA (+) SMP30/GNL KO mice. White arrows indicate congestion in capillary vessels. Two-directional white arrows indicate airspace in alveoli. Bars $=100 \mu \mathrm{m}$. (c) The intra-alveolar wall distance of the lung was assessed as mean linear intercepts (MLI) (indicated by two-directional white arrows in $\mathbf{a}$ and $\mathbf{b}$ ). Values are expressed as means \pm SEM of 10 animals. ${ }^{*} P<0.01$. $\mathrm{AA}$, ascorbic acid; $\mathrm{GNL}$, gluconolactonase; $\mathrm{KO}$, knockout; $\mathrm{PV}$, pulmonary vein.

sodium-dependent vitamin $\mathrm{C}$ transporter $2 \mathrm{KO}$ mice exhibited stunted neurite outgrowth in the absence of AA. Harrison et al. (21) additionally noted that AA depletion during prenatal and postnatal development led to elevations in oxidative stress in the brains of Gulo KO mice. However, those studies did not clarify whether the absence of AA during gestation affected other tissues during fetal and neonatal life.

Our morphological analysis of whole-body sections revealed tissue abnormalities in the heart, lungs, liver, and vertebral column of neonates from AA (Low) SMP30/GNL $\mathrm{KO}$ mice. In particular, the liver and lungs of these offspring manifested markedly severe congestion, presumably caused a
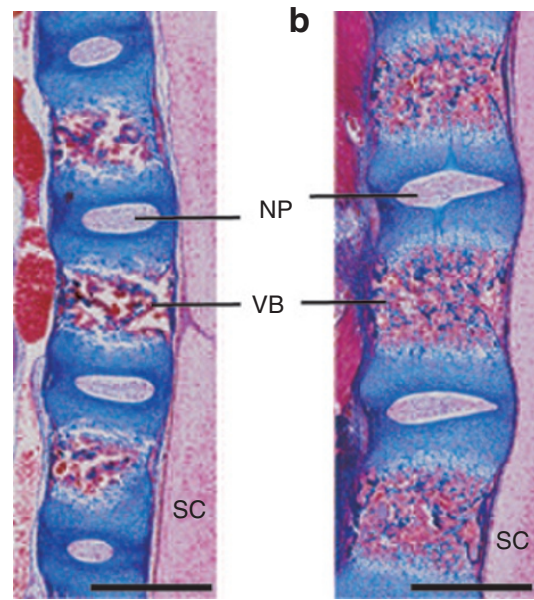

Figure 6. Histological analysis of sagittal sections of the thoracic vertebral column of neonates from (a) AA (Low) and (b) AA (+) SMP30/GNL KO mice. Bars $=100 \mu \mathrm{m}$. AA, ascorbic acid; GNL, gluconolactonase; KO, knockout; $\mathrm{NP}$, nucleus pulposus; SC, spinal cord; SMP, senescence marker protein; VB, vertebral bodies.

by some malfunction of the circulatory system. In fact, the hearts of these infant mice from AA (Low) SMP30/GNL KO mothers were abnormally enlarged. The myocardium was significantly thinner than that of the offspring from AA (+) SMP30/GNL KO mice, as documented here for the first time. Moreover, pulmonary alveoli were incompletely expanded in the neonates from AA (Low) SMP30/GNL KO mice. Similar results were observed in the lungs from sodium-dependent 
vitamin $\mathrm{C}$ transporter $2 \mathrm{KO}$ mice in which pulmonary alveoli failed to expand postnatally (19). These results suggest that a low intake of AA during the gestational period leads to congestive heart failure in neonatal mice. Still, direct physiological evidence for such cardiac failure is exiguous because the cause of this cardiac dilation might be impaired heart contractility and/or decreased resistance of internal blood pressure. However, Maeda et al. (12) also reported that an absence of AA led to aortic wall damage in Gulo KO mice. If so, a complete AA deficiency could cause the fragile heart of a fetal mouse to fail during the gestational period, although the mechanism of action is still unclear.

AA is also an essential cofactor in the hydroxylation of proline and lysine, which contribute to the stability of collagen's triple helix conformation $(6,7)$, act as electron donors, and reduce reactive oxygen species such as superoxide radicals and hydroxyl radicals $(8,9)$. Therefore, the cause of tissue abnormalities in neonates from AA (Low) SMP30/GNL KO mothers might be impaired collagen formation and/or a surfeit of oxidative stress. In fact, we previously reported that a complete lack of AA causes emphysema resulting from oxidative stress and a decrease of collagen synthesis in the lungs of SMP30/ GNL KO mice (22). Moreover, one reason why all fetuses deprived of AA died in utero could be a collagen deficiency, because colla1 KO mice, which cannot synthesize type 1 collagen, suffer from an embryonic lethal mutation (23).

In summary, we found that an absence or low intake of AA during pregnancy induced multiple abnormalities in the developing tissues of SMP30/GNL KO mice. Therefore, a diet that supplies an adequate amount of AA is essential to provide optimal conditions for fetal and neonatal health.

\section{METHODS}

\section{Animals}

SMP30/GNL KO mice were generated by the gene-targeting technique as described previously (24). Female SMP30/GNL KO (SMP30/GNL $\left.L^{-/-}\right)$ mice were mated with male SMP30/GNL KO $\left(S M P 30 / G N L^{Y /-}\right)$ mice to produce SMP30/GNL KO (male SMP30/GNL ${ }^{Y /-}$ and female SMP30/ $\left.G N L^{-/-}\right)$mice under normal AA conditions; that is, the mice had free access to water containing $1.5 \mathrm{~g} / \mathrm{l} \mathrm{AA}$ and $100 \mu \mathrm{mol} / \mathrm{l}$ EDTA. The concentration of AA in the drinking water was enough to maintain normal AA levels in all tissues (25). EDTA was added to stabilize AA in drinking water, which retained that stable state at least for $4 \mathrm{~d}$. Water bottles were changed every 3 or $4 \mathrm{~d}$. In addition, all mice were fed an AA-free diet (CL-2, CLEA Japan, Tokyo, Japan) ad libitum. Throughout the experiments, animals were maintained on a 12-h light/dark cycle in a controlled environment. All experimental procedures using laboratory animals were approved by the Animal Care and Use Committee of the Tokyo Metropolitan Institute of Gerontology.

\footnotetext{
Absent AA Conditions

Female SMP30/GNL KO (SMP30/GNL $\left.{ }^{-/-}\right)$mice deprived of any AA supplement were mated with male SMP30/GNL KO (SMP30/GNL $\left.L^{Y /-}\right)$ mice for $12 \mathrm{~h}$ and then checked for a vaginal plug to verify pregnancy. The vaginal plug-positive SMP30/GNL KO females were divided into two groups: normal AA (AA (+)) and absence of AA (AA (-)). The AA $(+)$ group had free access to water containing $1.5 \mathrm{~g} / \mathrm{l} \mathrm{AA}$ and $100 \mu \mathrm{mol} / \mathrm{l}$ EDTA, whereas the AA (-) group was given water without AA during the gestational period. To determine the AA level in the plasma, blood was collected from the tail vein every $5 \mathrm{~d}$ during gestation. A third group comprised SMP30/GNL KO (SMP30/GNL $\left.{ }^{-l-}\right)$ females divided before mating into normal AA (AA (+)) and low AA (AA (Low)) groups.
}

\section{Low AA Conditions}

Before mating, female SMP30/GNL KO mice were grouped as AA normal (AA $(+))$ or AA low (AA (Low)). The AA $(+)$ group received water with $1.5 \mathrm{~g} / \mathrm{l} \mathrm{AA}(100 \%$, normal AA), and the AA (Low) group received water with $0.0375 \mathrm{~g} / \mathrm{l} \mathrm{AA}(2.5 \%$, low AA) for $30 \mathrm{~d}$. During a 12-h period, both groups were then mated with SMP30/GNL KO $\left(S M P 30 / G N L^{Y /-}\right)$ males. The impregnated AA (+) and AA (Low) SMP30/GNL KO females (i.e., vaginal plug-positive) continuously received water containing $1.5 \mathrm{~g} / \mathrm{l}$ AA and $0.0375 \mathrm{~g} / \mathrm{l}$ AA containing $100 \mu \mathrm{mol} / \mathrm{l}$ EDTA, respectively, during the gestational period. Some fetuses of the AA (+) and AA (Low) SMP30/GNL KO mice were extirpated on day 19 of pregnancy. The tissues of interest were weighed and stored at $-80^{\circ} \mathrm{C}$ until use. Neonatal mice were collected within $24 \mathrm{~h}$ after birth. Some neonates of the AA (+) and AA (Low) SMP30/ GNL KO mice were maintained to evaluate each life span under the same AA conditions. The number of dead neonatal mice was counted.

\section{Morphological Analysis}

For whole-body analysis, the neonatal mice were killed, fixed in a $10 \%$ formalin neutral buffer solution (Wako Pure Chemicals, Osaka, Japan), embedded in paraffin, and sectioned into 4- $\mu \mathrm{m}$ thicknesses along the median line for histology. The sections were deparaffinized and then subjected to staining with hematoxylin and eosin, Elastica van Gieson, or Azan. Digital images of all specimens were captured using the virtual slide imaging system, VS100 (Olympus, Tokyo, Japan). For morphologic evaluation, uniform sagittal plane sections of the heart including the left atrium, left ventricular and left atrial appendages, pulmonary vein, aorta, and apex were prepared. The epicardial length of the heart and the thickness of the myocardium adjacent to the chest, apex, and back side in the left atrium were measured using WinROOF image analysis software (Mitani, Fukui, Japan). For morphologic evaluation of the lungs, the intra-alveolar wall distance was assessed by determining the mean linear intercepts according to the method described by Thurlbeck (26). More than 10 randomly selected fields in each section at $\times 100$ magnification were used for the calculation of mean linear intercepts.

\section{Measurement of AA}

AA was measured using high-performance liquid chromatography and electrochemical detection according to the methods described previously (27).

\section{Statistical Analysis}

The results are expressed as means \pm SEM. The probability of statistical differences between experimental groups was determined by one-way ANOVA followed by Dunnett and Tukey HSD post hoc comparisons and Student's $t$ test using KaleidaGraph software (Synergy Software, Reading, PA). Statistical differences were considered significant at $P<0.05$.

\section{ACKNOWLEDGMENTS}

We thank Phyllis Minick for the excellent English editorial assistance.

\section{STATEMENT OF FINANCIAL SUPPORT}

This work was supported by the Japan Society for the Promotion of Science KAKENHI Grant 24380073 to A.I. and 23590441 to N.M.

\section{REFERENCES}

1. Lane DJ, Lawen A. Ascorbate and plasma membrane electron transportenzymes vs efflux. Free Radic Biol Med 2009;47:485-95.

2. Eipper BA, Mains RE, Glembotski CC. Identification in pituitary tissue of a peptide alpha-amidation activity that acts on glycine-extended peptides and requires molecular oxygen, copper, and ascorbic acid. Proc Natl Acad Sci USA 1983;80:5144-8.

3. May V, Eipper BA. Regulation of peptide amidation in cultured pituitary cells. J Biol Chem 1985;260:16224-31.

4. Friedman S, Kaufman S. 3,4-dihydroxyphenylethylamine beta-hydroxylase. Physical properties, copper content, and role of copper in the catalytic activity. J Biol Chem 1965;240:4763-73. 


\section{Articles $\mid$ Kishimoto et al.}

5. Levine M, Morita K, Pollard H. Enhancement of norepinephrine biosynthesis by ascorbic acid in cultured bovine chromaffin cells. J Biol Chem 1985;260:12942-7.

6. Levine $\mathrm{M}$. New concepts in the biology and biochemistry of ascorbic acid. N Engl J Med 1986;314:892-902.

7. Peterkofsky B. Ascorbate requirement for hydroxylation and secretion of procollagen: relationship to inhibition of collagen synthesis in scurvy. Am J Clin Nutr 1991;54(Suppl 6):1135S-40S.

8. Nishikimi M. Oxidation of ascorbic acid with superoxide anion generated by the xanthine-xanthine oxidase system. Biochem Biophys Res Commun 1975;63:463-8.

9. Bielski BH, Richter HW, Chan PC. Some properties of the ascorbate free radical. Ann N Y Acad Sci 1975;258:231-7.

10. Nishikimi M, Koshizaka T, Ozawa T, Yagi K. Occurrence in humans and guinea pigs of the gene related to their missing enzyme L-gulonogamma-lactone oxidase. Arch Biochem Biophys 1988;267:842-6.

11. Mizushima Y, Harauchi T, Yoshizaki T, Makino S. A rat mutant unable to synthesize vitamin C. Experientia 1984;40:359-61.

12. Maeda N, Hagihara H, Nakata Y, Hiller S, Wilder J, Reddick R. Aortic wall damage in mice unable to synthesize ascorbic acid. Proc Natl Acad Sci USA 2000;97:841-6.

13. Gabbay KH, Bohren KM, Morello R, Bertin T, Liu J, Vogel P. Ascorbate synthesis pathway: dual role of ascorbate in bone homeostasis. J Biol Chem 2010;285:19510-20.

14. Ishigami A, Maruyama N. Significance of SMP30 in gerontology. Geriatr Gerontol Int 2007;7:316-25.

15. Maruyama N, Ishigami A, Kondo Y. Pathophysiological significance of senescence marker protein-30. Geriatr Gerontol Int 2010;10(Suppl 1): S88-98.

16. Kondo Y, Inai $Y$, Sato $Y$, et al. Senescence marker protein 30 functions as gluconolactonase in $\mathrm{L}$-ascorbic acid biosynthesis, and its knockout mice are prone to scurvy. Proc Natl Acad Sci USA 2006;103:5723-8.
17. Qiu S, Li L, Weeber EJ, May JM. Ascorbate transport by primary cultured neurons and its role in neuronal function and protection against excitotoxicity. J Neurosci Res 2007;85:1046-56.

18. Tveden-Nyborg P, Lykkesfeldt J. Does vitamin C deficiency result in impaired brain development in infants? Redox Rep 2009;14:2-6.

19. Sotiriou S, Gispert S, Cheng J, et al. Ascorbic-acid transporter Slc23a1 is essential for vitamin $C$ transport into the brain and for perinatal survival. Nat Med 2002;8:514-7.

20. Adlard BP, De Souza SW, Moon S. Ascorbic acid in fetal human brain. Arch Dis Child 1974;49:278-82.

21. Harrison FE, Meredith ME, Dawes SM, Saskowski JL, May JM. Low ascorbic acid and increased oxidative stress in gulo(-/-) mice during development. Brain Res 2010;1349:143-52.

22. Koike K, Kondo Y, Sekiya M, et al. Complete lack of vitamin C intake generates pulmonary emphysema in senescence marker protein-30 knockout mice. Am J Physiol Lung Cell Mol Physiol 2010;298:L784-92.

23. Schnieke A, Harbers K, Jaenisch R. Embryonic lethal mutation in mice induced by retrovirus insertion into the alpha 1 (I) collagen gene. Nature 1983;304:315-20.

24. Ishigami A, Fujita T, Handa S, et al. Senescence marker protein-30 knockout mouse liver is highly susceptible to tumor necrosis factor-alpha- and Fas-mediated apoptosis. Am J Pathol 2002;161:1273-81.

25. Iwama M, Shimokado K, Maruyama N, Ishigami A. Time course of vitamin C distribution and absorption after oral administration in SMP30/ GNL knockout mice. Nutrition 2011;27:471-8.

26. Thurlbeck WM. The internal surface area of nonemphysematous lungs. Am Rev Respir Dis 1967;95:765-73.

27. Sato Y, Uchiki T, Iwama M, Kishimoto Y, Takahashi R, Ishigami A. Determination of dehydroascorbic acid in mouse tissues and plasma by using tris(2-carboxyethyl)phosphine hydrochloride as reductant in metaphosphoric acid/ethylenediaminetetraacetic acid solution. Biol Pharm Bull 2010;33:364-9. 